\title{
Identify urban spatial patterns based on the plot shapes and building setting in downtown of Nanjing
}

\author{
Yusheng Gu, Lian Tang, Wowo Ding* \\ School of Architecture and Urban Planning, Nanjing University, Nanjing, China \\ *(corresponding author) \\ E-mail: guyushengnju@163.com, tanglian@nju.edu.cn,dww@nju.edu.cn
}

\begin{abstract}
The geometric characteristics of modern cities have been difficult to describe that is important for urban design, which deserve to be further interpreted. Taking advantage of Conzen's methodology, the building is tightly related with its plot, which means the certain building pattern can be described by examining the generation of the building arrangement within the plot. Simultaneously, the building pattern is highly affected by the plot pattern it is located. In view of these, plot patterns together with land property and site coding could be taken as the clue for understanding both building patterns and urban spatial configuration. 35 commercial blocks in Nanjing downtown areas are chosen as research samples. Firstly, the internal structure of the blocks will be studied by analyzing the patterns, functions and land utilities of its plots. Focusing on the site coding and regulation, the building arrangement could be clarified and mapped. The results will identify the urban spatial patterns in downtown of Nanjing by mapping the characteristics of plot size, shape, properties and boundary lines. Therefore, the method on describing urban spatial configuration in modern cities could be developed.
\end{abstract}

Keywords: Spatial Configuration, Building Pattern, Plot Pattern, Plot Boundary Line

\section{Introduction}

With the further development of reform and opening-up, China has experienced rapid urbanization and the physical space of many cities has undergone tremendous changes. As one of the most developed cities in China, Nanjing has a dramatic scale expansion and significant changes in material form. The study of urban morphology is concerned with the material form of the city. And the urban form is the materialized result of the development of urban space. The geometric characteristics of modern Chinese cities such as Nanjing are complex and difficult to describe. The urban spatial form is enclosed by buildings and has a figure-ground relationship with the urban texture. The study of the spatial form could provide new perspectives and methods for the study of urban morphology. Many studies have shown that the spatial form of the city has an important influence on the visual and inner perception. And it is also associated with the wind environment and the thermal environment which is link to health of the public. Therefore, the study of the spatial form especially to make the spatial form descriptive is of great value.

The study of urban morphology contains many different disciplines, and scholars in different fields have different perspectives. But both geographers and architects can come to the conclusion that the smallest unit of the city is the union of the plot and the buildings it contains. Take Nanjing as an example, the roads divide the city into blocks and each block is made up of several or even dozens of plots. Each building is generated in its corresponding plot although it may have different layout patterns and shapes due to the requirements of the owner and the designer's aesthetic. What's 

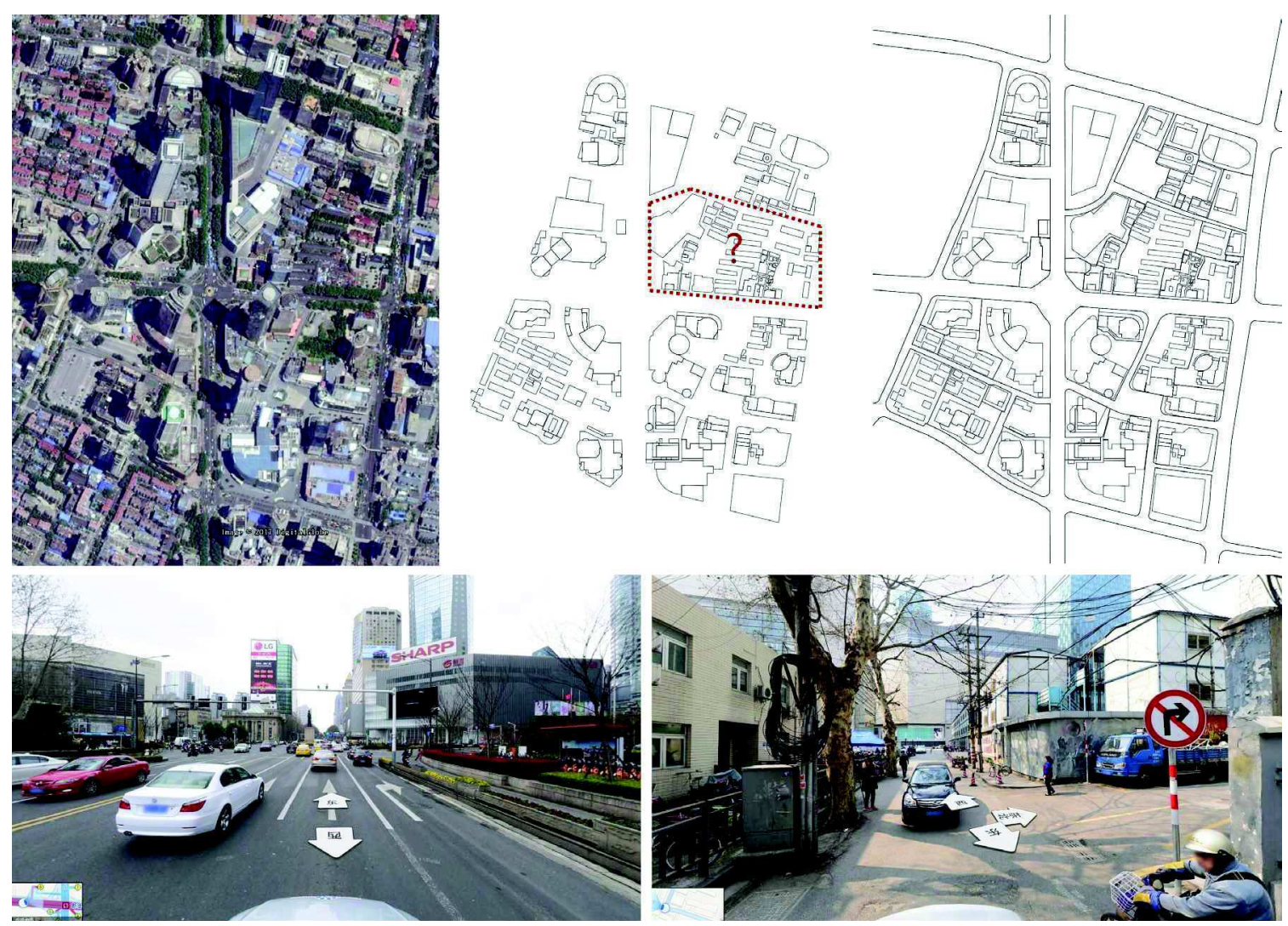

Figure 1.

Four Blocks in City Center of Nanjing

more, the building design must meet the design specifications. Thus the roads and the plot boundaries can be used as a tool for studying the urban spatial form enclosed by the vertical façade of the buildings.

What we need to study is the spatial form between the buildings in the city. One is the spatial form enclosed by buildings on both sides of urban roads, and the other is the spatial form enclosed by buildings between the adjacent plots. Taking the high-density area in the center of Nanjing as an example, the above two kinds of spatial form are studied. Urban roads are mainly divided into four types: expressway, main road, secondary road and branch road. The plots are divided into residential plots and commercial plots from the land use. The spatial pattern of residential plots is already clear and its texture has been quantified. The internal spatial form of residential plots is mainly determined by the sunshine demand and the required volume ratio. However, there are no definite organization rules in the shape and the texture of the buildings in commercial plots. So the research will focus on the space form between commercial plots and adjacent plots.

This paper will focus on the following aspects: for the spatial form enclosed by buildings along roads, this paper will analyze the building concession to the road redline and the layout of the entrance and exit of the building, so that the general rules of the formation of the spatial form can be made. For the spatial form enclosed by buildings between adjacent plots, the plot boundary lines can be used as a tool to summarize, analyze and describe it.

\section{Research and Analysis}

The study chooses four blocks in XinJieKou which is the city center of Nanjing as a case (See figure 1). The urban texture of this four blocks is rich and varied, which can be regarded as a microcosm of the complex texture of modern Chinese cities. Firstly, a complete general plan of the central area is drawn based on the satellite map from 2005 to 2017. If you 

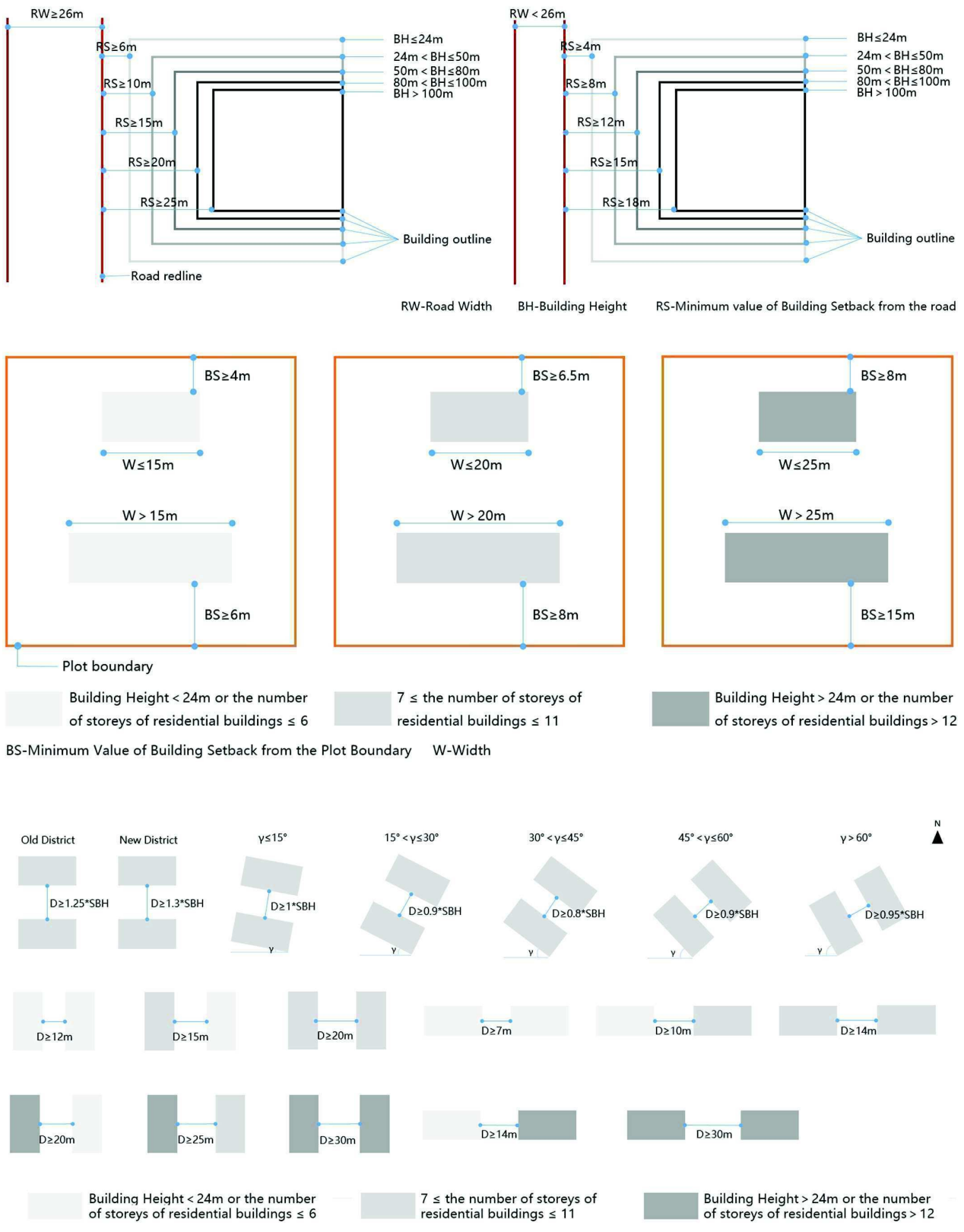

Figure 2.

Diagram the $<$ Implementing Rules of Nanjing City Planning Regulations $>, 2004$

only keep the buildings in the central area, you will find that the spatial form is disorganized and hard to describe. But if you join the city roads and the plot boundary lines in which the building is located, the situation will be different. Combing the road network system to clarify the blocks and the plot groups. Then to clear the plot land use into commercial plots and residential plots. Because the design code of Nanjing is revised and improved over the years, it is necessary to find out the construction date each plot and then to find the design specifications of that year for verification and research. Thus, the analysis of the spatial form between buildings can be found regular.

\section{Research on laws and regulations}

Laws and regulations for building patterns mainly include two aspects: the first is the building concession to lines, including road 

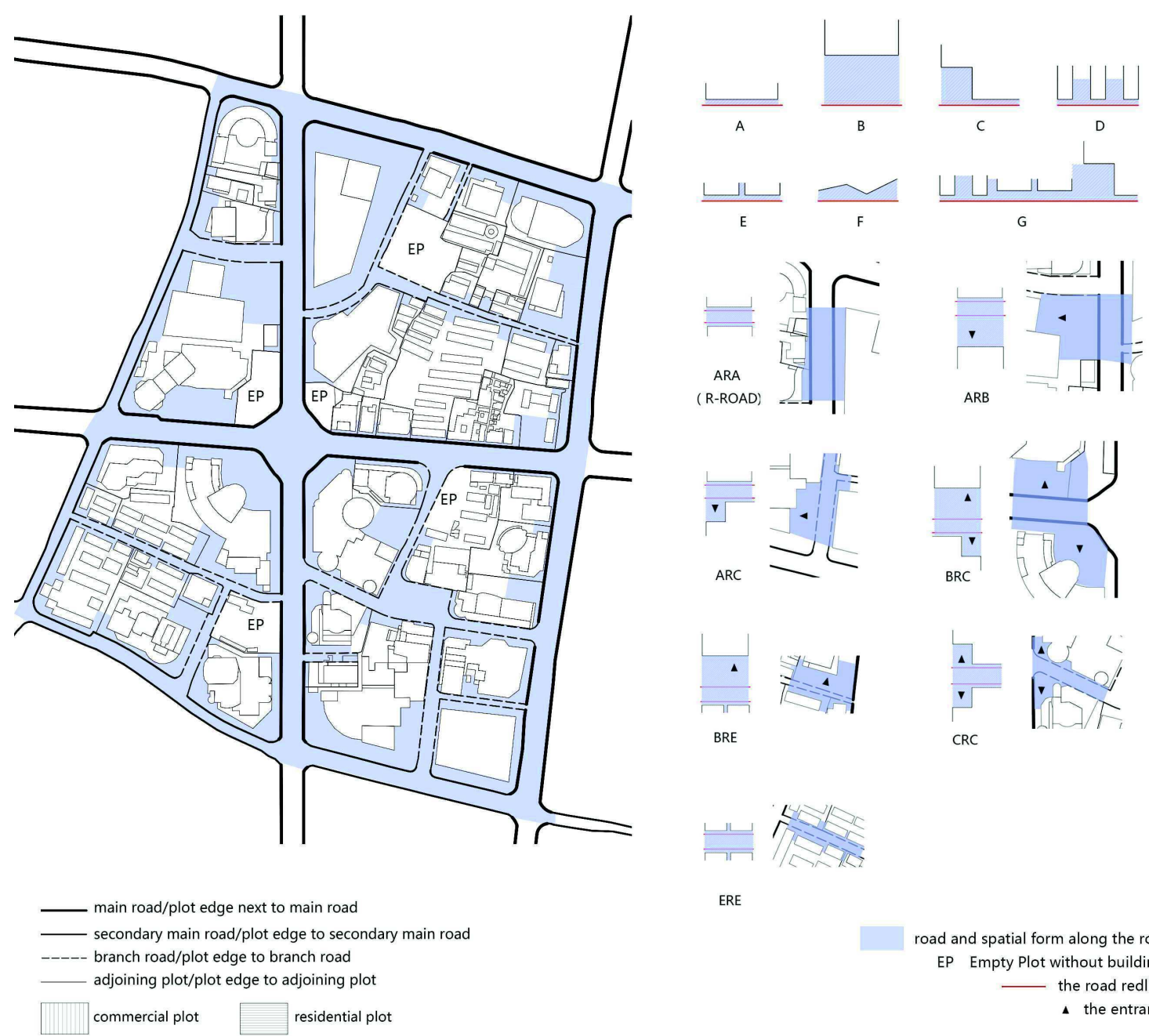

ERE

road and spatial form along the road EP Empty Plot without buildings the road redline

Figure 3.

Spatial Form between Buildings Adjacent to the Road

redlines and plot boundary lines. The second is the required spacing between buildings, which is mainly to meet the sunshine demand and the fire demand.

The building concession to lines is to limit the minimum distance from the building outline to the road redline or the plot boundary line. The concession distance to road redline is linked to the road level and the height of the building. The wider the road is, the higher the building is, the concession distance will be greater. The concession distance to plot boundary line is related with the height and width of the building. The higher the building is, the larger the width is, the concession distance will be greater. The concession distance between buildings especially between residential buildings must meet the sunshine demand and fire demand. And the specification makes different arrangements for parallel layout, vertical layout and slant layout of buildings. Taking the $<$ Implementing Rules of Nanjing City Planning Regulations $>(2004)$ as an example, the requirements are diagramed in Figure 2.

The fire requirements on the spacing between buildings are related with building height and building fire rating. Taking the $<$ Code for Fire Protection Design of Tall Buildings $>$ (2006) as an example, the Table 1 sets out the minimum fire spacing distance between buildings.

\section{Research on Spatial Form}

Spatial Form between Buildings Adjacent to the Road

The impact of urban roads on city layout is often very enduring. And most of the historical 

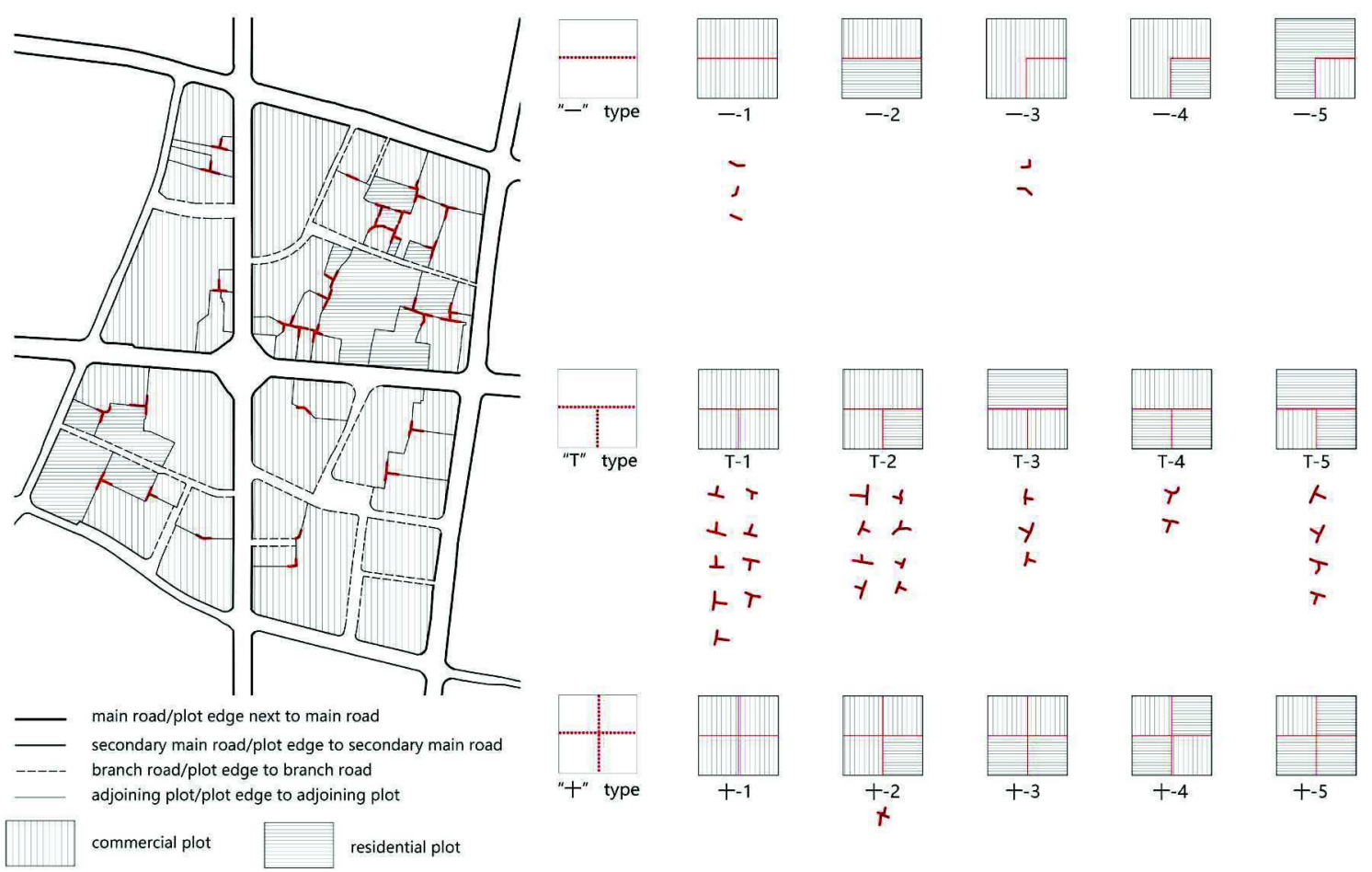

Figure 4.

Combinations of Plots in City Center of Nanjing

roads will be repaired or widened to continue to be used. In the new district of the city, the road network system is planned first and blocks are divided by this system. Therefore, when studying the spatial form of modern Chinese cities like Nanjing, the first consideration is the spatial form enclosed by buildings on both sides of roads.

After combining the requirements of the specification and case study, it could be found that the spatial form between the building and the adjacent road can be divided into two categories: the first kind is to spare a road which can be used as the fire channel. The second kind is to spare a square used as entrance, parking or landscape in the corner of the building or the whole building. Considering the nature of the plot where the building is located, there are seven types of spatial form between the building and the road (See Figure 3). The first is the road space(A) formed by building concession to the road. The second is the square space(B) formed by building concession to the road. The third is the corner square $\operatorname{space}(\mathrm{C})$ formed for that the building cuts out the entrance space in the corner. The fourth is the jagged space(D) formed when the north-south road is adjacent to residential plot. The fifth is the T-shape space(E) formed when the east-west road is adjacent to residential plot. The sixth is the irregular space(F) formed because of the building shape, which can be used as road, square or parking. The seventh is the combination of two or more of the first six types $(G)$. The spatial form between the buildings adjacent to the road is combination of the seven types above and the road itself. Theoretically, there are $7 * 7=49$ species of spatial form between the buildings adjacent to the road. And these species may combine with each other along the road, so the spatial form along the road could be very complex. But each type of spatial form can be explained.

Take the central area of Nanjing as an example, there are seven typical spatial forms along the road (See Figure 3). The spatial form could be named by the spatial form of one side + road + the spatial form of the other side, so the seven types can be named as ARA, ARB, ARC, BRC, CRC, ERE. Thus, it is possible to use this numbering method to address and describe the spatial forms enclosed by buildings along city roads. 


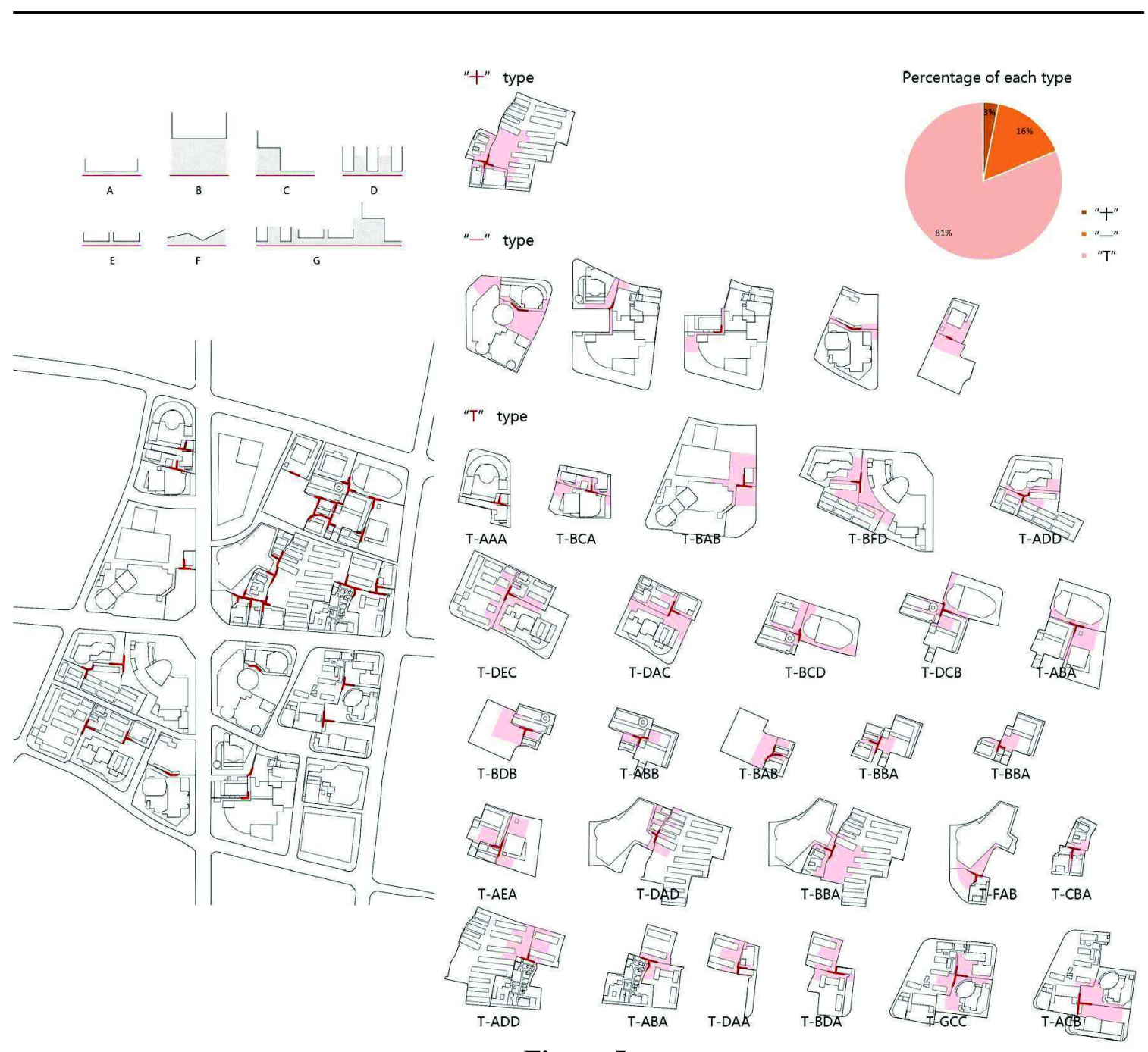

Figure 5.

Spatial Form between Adjacent Plots

Spatial Form between Adjacent Plots

In addition to the spatial form between buildings adjacent to the road, the spatial form between adjacent plots is also worth considering. For that the buildings in the city are grown in the plots, their external spatial form is closely related to the plot boundary. Thus the plot boundary line could be used as the tool to study the spatial form.

There are three kinds of combinations of plots (See Figure 4). The first kind is the ".." type which means two plots have one side intersecting. The second kind is the " $\mathrm{T}$ " type which means three plots intersect to each other. The third kind is the "+" type which means four plots present a cross shape. Take the city center of Nanjing as an example, there are five "-"-type plots (accounting for 15.6\%) and twenty-six " $T$ "-type plots (accounting for
81.3\%) and only one "+"-type plot (accounting for $3.1 \%$ ). The paper focuses on the spatial form associated with the commercial plots and will not discuss the situation when all combined plots are residential plots. According to the land use of different plots, every one of the three types has five different combinations (See Figure 4). Numbering them we can get from "-"-1 to "-"-5, from "T"-1 to " $T$ "- 5 , from " +"-1 to "+"-5, totaling fifteen numbered types. Statistics on the number of each type among thirty-three blocks in Nanjing are as follows. It can be found that both "-" type and "+" type are few and " $T$ " type is the main type, especially T-4 and T-5 are the largest in number.

Taking the central area of Nanjing as an example, the spatial form adjacent to the plot boundary line is drawn as shown in Figure 5. The number of "+" type is very few. The number of "-" type is few and its spatial form can be 
considered as the case of spatial form along the road when the road width is zero. Therefore, the study will focus on "T" type. There are three main types of space form in "T" type: the first type is the bar space which can satisfy the traffic and fire demand. The second type is the square space which is a partial or whole concession of the building to the plot boundary to form the entrance or parking space. The third type is the space formed by plot boundary and residential buildings. The spatial forms of "T" type are made up of the space above. And the space can also be abstracted into the $\mathrm{A}$ to $\mathrm{G}$ type in Figure 4. So all the spatial forms can be numbered and described.

\section{Conclusion}

Urban texture of modern cities is complex and is difficult to be described. However, the description can be made from the perspective of spatial form between buildings. The city road and the plot boundary line can be the tool for the study. The spatial form is divided into the spatial form along the road and the spatial form between adjacent plots. The design code has regulations for building concession to the road or to the plot boundary. It also defines the minimum distance between buildings for sunshine demand or fire demand. As a result, the design code has great guiding significance for the formation of the spatial form.

The spatial form between the building and the road can be abstracted and simplified into seven types and the spatial from along roads can be numbered and described. The spatial form between adjacent plots are closely related to the combination patterns of the plots. The "-." type, the "T" type and the "+" type are common types, among which " $T$ " type accounts for the vast majority. The study of the central area of
Nanjing shows that the spatial form between plots is complex and varied, but the basic types are the same which means the spatial form can also be numbered and described.

\section{Acknowledgements}

This research is funded by National Natural Science Foundation of China (No. 51538005).

\section{References}

Conzen, M.R.G. (1960) "Alnwick, Northumberland: A Study in Town Plan Analysis", Institute of British Geographers.

Conzen, Michael P. (2004) Thinking about urban form : papers on urban morphology, Peter Lang Publishing.

Dongxue Wang (2016) The relationship between the space of block and the plot boundary-based on a general survey in Nanjing, Master's thesis of Nanjing University.

Jingjing Jiang (2015) Commercial plots and building patterns analysis-based on a general survey in Nanjing, Master's thesis of Nanjing University.

Gianfranco Caniggia, Gian Luigi Maffei(2001) Architectural Composition and Building Typology: Interpreting Basic Building, Firenze: Aliena.

Lina Zhang (2013) Urban Plot Characteristics Study: Casting Center District in Nanjing, China, International Seminal of Urban Morphology.

J.W.R.Whitehand (2011) Issues in Urban Morphology, Urban Morphology.

Karl Kropf (2009) Aspects of Urban Form, Urban Morphology.

\section{Appendix}

Table 1

\begin{tabular}{ccccccc|}
$\begin{array}{c}\text { Building } \\
\text { type }\end{array}$ & tower & podium & \multicolumn{4}{c|}{ Other civil building } \\
\cline { 3 - 6 } & & & First and second level & Third level & Fourth lever \\
\hline tower & 13 & 9 & 9 & 11 & 14 \\
\hline podium & 9 & 6 & 6 & 7 & 9 \\
\hline
\end{tabular}

